\title{
The Physical Nature of Velocity
}

\author{
Zhonggang $\mathrm{Li}^{1}$ \\ 1 Technology Department, Silkworm Research Institute, Beiheishan Village, Dasheng Town, Anqiu City, \\ Shandong Province, 262128, China \\ Correspondence: Zhonggang Li, Technology Department, Silkworm Research Institute, Beiheishan Village, \\ Dasheng Town, Anqiu City, Shandong Province, 262128, China. E-mail: 13465701349@163.com
}

Received: August 10, 2018

Accepted: October 20, 2018

Online Published: November 30, 2018

doi:10.5539/apr.v10n6p15

URL: https://doi.org/10.5539/apr.v10n6p15

\begin{abstract}
Matter and energy are made up of the same basic particles. Why, then, is there a significant difference between matter and energy? This is because their basic particle compositions differ. The basic particle is the basic unit of mass and energy. Mass and energy conservations are essentially basic particle conversions. The basic particle is a vector, moving at the maximum velocity of the universe; however, after a substance tangibly solidifies, this velocity becomes zero. The velocity of a moving object is, thus, the ratio between the basic particles contributing to energy and those contributing to mass, and the direction of its velocity is determined by the basic particle directions. Electrons, photons, neutrons, protons, neutrinos, and other microscopic particles consist of basic particles. The total mass of a moving body increases with increasing velocity. This added mass is composed of basic particles provided by an external system. As relativity is a mathematical model, its equations may satisfy mathematical principles even though some of them may not represent objective physical facts; instead, these may simply be mathematical solutions without physical meanings.
\end{abstract}

Keywords: Mass; energy; velocity; concentration; reference system

\section{Introduction}

Disagreements over many physics questions can be attributed to differences in individual interpretations of the fundamentals of physics, creating some diametrically opposing views based on the same concepts. Consequently, there are serious differences between fellow researchers attempting to answer questions using different approaches. Under these circumstances, wherein no approach appears successful, it becomes especially necessary to revisit the fundamentals of physics. Could it be that the bases of these approaches were incorrect?

It can be agreed upon that modern physics is quite profound and complex. However, it is unclear if this is because the world is complex or if this complexity was created by the use of complex physics using countless theories and symbols to describe the world because of an inability to comprehend the world as it realistically is. In this context, the possibility must be considered that physicists are over-thinking concepts, making the world appear more complex than it truly is. To address this consideration, return to the origins of physics.

Modern physics is founded on the concepts of displacement and time, which have been used to describe concepts such as velocity and acceleration. Many theories in modern physics were progressively constructed from these strictly defined concepts: the concept of mass is closely related to the concepts of displacement and time; and the fundamental concepts of physics, such as displacement, time, mass, and energy, are involved in the concept of velocity. This article thus focuses on velocity.

Since the nineteenth century, new problems have appeared as physics has developed. For example, the speed of light remains unchanged regardless of the light source or the relative motion of the observer. This contradicts the principle of velocity in classic physics theory. Einstein suggested the theory of special relativity to address this scenario at the beginning of the twentieth century, presenting the concept of relative space-time and fundamentally altering the concepts of both time and space. The theory of relativity references that the length and mass of an object are related to its speed. The theory of relativity, established by the deductive method, solved many problems; however, it also introduced new problems. The question thus arises: is the theory of relativity the only choice for physics? To answer this, it is necessary to start from the basic concepts of physics, and rethink their basic problems. As many physical problems relate to speed, this necessitates a study of the concept of speed. 


\section{Derivation and Calculations}

Mechanical motion, which can be fast or slow, is the most common physical phenomenon in the universe. In physics, the concept of velocity is used to describe motion. As velocity is a physical concept humans came to understand quite early on, it has undergone significant developments and improvements over the years. In modern physics, velocity is a physical quantity describing the speed and direction of motion in a point mass, and contemporary physicists describe it only in terms of displacement and time. Velocity is a relative quantity equal to the first derivative of displacement with respect to time and the time integral of acceleration. However, this ratio of displacement to time is simply a calculation method that fails to reflect the true nature of motion.

Since displacement and time are the only tools used to describe velocity, it is necessary to ask: what is the true nature of velocity? For hundreds of years, it has been considered unnecessary to ponder this any further. However, it may be possible to describe velocity without considering displacement and time. Because the concepts of displacement and time form the foundations of modern physics, they have been used to describe concepts such as velocity and acceleration which many theories in modern physics have been built upon. The concept of mass is closely related to the concepts of displacement and time. The definition of mass in modern physics, built on Mach's criticism of Newton's definition of mass (Mach, 1907).

), uses a recursive definition method. Using the international prototype of kilogram (IPK) to define one kilogram, the mass of an unknown object is equal to the ratio of acceleration of one IPK to acceleration of the object. This methodology determines an accurate numerical value for the mass of the object in question, demonstrating that the concept of mass in modern physics is derived from displacement and time. Further consider the concept of energy. In the book Feynman's Lectures on Physics (Feynman, 1982), energy is defined as follows:

"There is a certain quantity, which we call energy, that does not change in the manifold changes which nature undergoes. That is the most abstract idea, because it is a mathematical principle; it says that there is a numerical quantity, which does not change when something happens. It is not a description of a mechanism, or anything concrete; it is just a strange fact that we can calculate some number and when we finish watching nature go through her tricks and calculate the number again, it is the same."

This implies that energy in modern physics is simply a numerical value calculated using special methods. If the related quantities - displacement, time, and mass - have not first been measured, the numerical value of energy cannot be derived. At this interval, the possibility of constructing a reliable physics model and deriving a meaningful formula for velocity using only the concept of mass must be considered.

As many concepts in physics were developed only recently, the constraints of modern concepts may be set aside by returning to the ideas of a few hundred years ago. This allows the consideration of velocity using only mass. (Here, an important hypothesis is postulated. Matter and energy consist of the same basic particles. Why, then, is there a significant difference between matter and energy? It is because the combination of these basic particles is not equal. The magnitude and direction of the velocity of a moving object are determined by such basic particles. Mass and energy can be used for addition and subtraction operations, essentially considering the quantity of basic particles. This produces $(m+Q)$. If the calculation in this paper is consistent with these facts, then, the above hypothesis is correct.) Imagine there is an object moving with velocity $V$. Its stationary mass is $m$, and its kinetic energy is $Q$. Assume for the moment that mass, matter, and energy are, by nature, equivalent. The body's velocity $V$ may then be considered to be the ratio of its kinetic energy $Q$ and total mass $(m+Q)$, similar to the concept of concentration. In non-technical language, the kinetic energy is equivalent to a solute, the stationary mass is equivalent to a solvent, and the velocity is the character of a solution in which mass and kinetic energy are combined. The magnitude of velocity is equal to the concentration of the solution, making velocity a property that an object has after it obtains kinetic energy, unrelated to any other factors. As the kinetic energy begins to account for more of the total mass, the velocity also increases. If kinetic energy accounts for only a small portion of the total mass, the velocity remains small.

This article is based on the basic particle considered from a new perspective, and the essence of further research regarding physical problems, this article, and all the physical laws in modern physics are consistent, not contradictory. These ideas are postulated with the desire to promote the further development of physics. There are many areas for addition and later improvement in the ideas and perspective proposed herein. From this perspective, the meanings of some physical concepts are slightly altered from their original ones.

The proposed formula is as follows: 


$$
V=\frac{Q}{m+Q} \cdot X
$$

where $X$ is a coefficient.

This is similar to the formula calculating the concentration of a solution. The percentage concentration of a soluble substance in a solvent forming a solution (the mass fraction of the solution), known as the solution percentage concentration, is expressed as:

$$
\text { Concentration } \cdot C=\frac{m(\text { solute })}{M(\text { solvent })+m(\text { solute })} \times 100 \%
$$

Removing the definitions, this is written as:

$$
C=\frac{m}{M+m} \times 100 \%
$$

Considering that this cannot be a simple ratio, we need a coefficient $X$. We can see that the concepts of time and displacement are not used, and the mathematical formula only involves a kind of ratio of masses, or a numerical ratio of some basic quantities. Considering the concentration analogy, when there is only solvent and no solute, the concentration is zero. When there is a solute but no solvent, the concentration reaches its maximum value of 1. Therefore, this velocity formula must satisfy the following conditions: when the object has no kinetic energy, the velocity is zero; when there is no resting mass but only energy, the velocity reaches its maximum value of 1 . In mathematical terms, the velocity formula must satisfy the following three strict requirements:

1) When $m \neq 0, Q=0, V=0$.

2) When $m=0, Q>0, V=1$, the velocity is at a maximum value of 1 .

3) When $m \neq 0, Q>0, V$ increases with $Q$, but never reaches 1 ; $V$ decreases with $Q$ but is always greater than zero. That is, $0<\mathrm{V}<1$.

The derivation of the following formulas is simple:

$$
\begin{aligned}
V & =\frac{Q}{m+Q} \cdot X \\
& =\sqrt{\frac{Q^{2} X^{2}}{(m+Q)^{2}}} \\
& =\sqrt{\frac{Q^{2} X^{2}}{m^{2}+2 m Q+Q^{2}}}
\end{aligned}
$$

From the derivation, we can see that the formula can meet all three of the above requirements only when $Q^{2} X^{2}=2 m Q+Q^{2}$. Therefore,

$$
\begin{aligned}
Q^{2} X^{2} & =2 m Q+Q^{2} \\
X^{2} & =\frac{2 m Q+Q^{2}}{Q^{2}} \\
X^{2} & =\frac{2 m}{Q}+1 \\
X & =\sqrt{\frac{2 m}{Q}+1}
\end{aligned}
$$

Therefore, the velocity formula can be expressed as follows:

$$
V=\frac{Q}{m+Q} \cdot \sqrt{\frac{2 m}{Q}+1}
$$


Other equivalent forms of the above equation include

$$
\begin{gathered}
V=\sqrt{\frac{2 m Q+Q^{2}}{m^{2}+2 m Q+Q^{2}}} \\
V=\sqrt{\frac{2 Q+\frac{Q^{2}}{m}}{m+2 Q+\frac{Q^{2}}{m}}} \\
V=\sqrt{\frac{Q(M+m)}{M^{2}}} \text { (where } M=m+Q \text { ) }
\end{gathered}
$$

If we assume that the maximum possible speed of 1 is the speed of light, all applications and calculations show that the results from this extremely simple starting point - the velocity equation - are completely consistent with reality. Remarkably, the results are consistent with relativity-based calculations! Whereas relativity-based formulas are derived from complicated space-time relationships, our formula was derived simply and intuitively by considering the concentration of a solution. Although the starting points are different, the calculated results are the same. The following simple comparison calculations verify this fact:

1) Assume an object with high velocity. When $M=1.7 \mathrm{~m}$ and the object's resting mass $m=1$, so that the additional mass of 0.7 comes from $Q$, what is the velocity? According to relativity-based calculations, $V \approx 0.8 c$, that is, $80 \%$ of the speed of light.

Using the velocity equation presented above, the result is:

$$
\begin{aligned}
& V=\frac{Q}{m+Q} \cdot \sqrt{\frac{2 m}{Q}+1} \\
& =\frac{7}{17} \times \sqrt{\frac{27}{7}} \\
& \approx 0.8(\text { or } 0.8 c)
\end{aligned}
$$

Hence, the results are the same.

Assuming that the maximum value of 1 is equal to the speed of light, if $V \approx 0.8$ and we use $3 \times 10^{8} \mathrm{~m} \cdot \mathrm{s}^{-1}$ as the speed of light, then $V \approx 2.4 \times 10^{8} \mathrm{~m} \cdot \mathrm{s}^{-1}$. The magnitude of $V$ depends on the specific units we use, which are arbitrary. The units only exist as tools that people use for convenience.

2) Assume an object moving at high velocity. When $\mathrm{M}=1.25 \mathrm{~m}$, what is the velocity? Using relativity-based formulas, we can arrive at $\mathrm{V}=0.6 \mathrm{c}$.

Using the velocity equation presented above, we get

$$
\begin{aligned}
& V=\frac{Q}{m+Q} \cdot \sqrt{\frac{2 m}{Q}+1} \\
& =\frac{1}{5} \times \sqrt{9} \\
& =0.6(\text { or } 0.6 c)
\end{aligned}
$$

We can see that the results of the two calculations are the same.

We can see that matter and energy are equivalent; then the velocity formula presented in this paper only expresses the proportional relationship between basic quantum quantities. Only by setting the velocity of 1 equal to some specific value, the formula gives us values corresponding to real-world units. For convenience, we can make substitutions in common concepts from modern physics. In this theory, matter and energy are equivalent, and are composed of the same type of basic particles. Therefore, I have set a temporary rule; the standard units of mass and energy are the same, i.e., kilogram, and the maximum speed is 1 . In essence, speed is a ratio, and it 
does not have units, but a maximum value of 1 , which is the speed of light. Hence, the calculation can give us values corresponding to real world units. The unit conversion is in full compliance with existing mathematical and physical laws.

Now, let us derive a formula for energy (we already know that $M=m+Q$ ).

According to the velocity formula

$$
V=\frac{Q}{m+Q} \cdot \sqrt{\frac{2 m}{Q}+1}
$$

Therefore,

$$
\begin{aligned}
& V^{2}=\frac{Q^{2}}{M^{2}} \bullet \frac{2 m+Q}{Q} \\
& M^{2} V^{2}=Q(2 m+Q) \\
& M^{2} V^{2}=Q(M+m)
\end{aligned}
$$

Thus, the energy formula is

$$
Q=\frac{M V^{2}}{1+\frac{m}{M}}
$$

We can draw the following three conclusions:

1) Under low-speed conditions, $M \approx m$. That is, $1+\frac{m}{M} \approx 2$. Therefore,

$$
\begin{gathered}
Q=\frac{M V^{2}}{1+\frac{m}{M}} \\
Q \approx \frac{1}{2} M V^{2}
\end{gathered}
$$

Here, we were able to derive the classical physics formula for kinetic energy without relying on the concepts of displacement or time. We can also see that this formula only applies to low-velocity conditions. The formula for kinetic energy in classical physics is based on a mechanical calculation. However, classical physics was developed to describe a low-velocity world, where this formula is accurate enough and its practical importance cannot be discounted.

The calculation result of this formula is consistent with the facts in reality. For example, for an object with mass of $1 \mathrm{~kg}$ in straight-line motion, if the speed is $\sqrt{2} \mathrm{~m} / \mathrm{s}$, what is the kinetic energy? The formula can be used to obtain the solution, as follows:

$$
\begin{aligned}
Q & =\frac{M V^{2}}{1+\frac{m}{M}} \\
Q & \approx \frac{1}{2} M V^{2} \\
& =\frac{1}{2} \times 1 \times \frac{2}{c^{2}} \\
& =\frac{1}{c^{2}} \mathrm{~kg}
\end{aligned}
$$


By using the kinetic energy formula of classical physics, the solution can be obtained:

$$
\begin{aligned}
& \mathrm{E}=\frac{1}{2} \mathrm{~m} \mathcal{V}^{2} \\
& =1 \mathrm{~J}
\end{aligned}
$$

According to the relativistic mass-energy equation, $\frac{1}{c^{2}} \mathrm{~kg}$ is equal to $1 \mathrm{~J}$; hence, the calculation result of this formula is consistent with the result of classical physics.

$$
\begin{aligned}
& \mathrm{E}=\mathrm{m}_{C^{2}} \\
& =\frac{1}{c^{2}} \times c^{2}=1 \mathrm{~J}
\end{aligned}
$$

2) Under high-speed conditions, we must consider the effects of changing mass. Thus, we get a dynamic energy equation, not a mechanical one.

$$
Q=\frac{M V^{2}}{1+\frac{m}{M}}
$$

3) When $m=0$, and $M \neq 0,1+\frac{m}{M}=1$. Therefore,

$$
Q=\frac{M V^{2}}{1+\frac{m}{M}}=M V^{2} \quad \text { and } \quad Q=M c^{2}
$$

We can see that the derived energy equation is equivalent to the mass-energy equation of relativity.

During the application of the energy equation, if we assume that the highest possible speed of 1 is equal to the speed of light (with its units), then the unit for both mass and energy is the kilogram. However, a kilogram represents an enormous amount of energy. The earth receives approximately $2 \mathrm{~kg}$ of energy from solar radiation every second, which is enough to drive many natural phenomena and sustain all life on earth. Therefore, the kilogram is not an appropriate unit for discussing the quantities of energy we encounter in daily life. If we use $\mathrm{m} \cdot \mathrm{s}^{-1}$ as the unit for velocity, then the unit for energy is Joule, which is more convenient to use. In essence, the specific units we use for energy depend on the number of parts into which we divide the maximum velocity.

\subsection{Verification of Formula}

Using the velocity formula derived above, we can also derive another energy formula. Using another form of the velocity equation from above:

$$
\begin{aligned}
& V=\sqrt{\frac{2 m Q+Q^{2}}{(m+Q)^{2}}} \\
& V^{2}=\frac{2 m Q+Q^{2}}{(m+Q)^{2}} \\
& m^{2} V^{2}+2 m Q V^{2}+Q^{2} V^{2}=2 m Q+Q^{2} \\
& \left(V^{2}-1\right) Q^{2}+\left(2 m V^{2}-2 m\right) Q+m^{2} V^{2}=0 \\
& Q=\frac{-b \pm \sqrt{b^{2}-4 a c}}{2 a}
\end{aligned}
$$




$$
\begin{aligned}
& =\frac{-\left(2 m V^{2}-2 m\right) \pm \sqrt{\left(2 m V^{2}-2 m\right)^{2}-4\left(V^{2}-1\right) m^{2} V^{2}}}{2\left(V^{2}-1\right)} \\
& =-m \pm \frac{2 m \sqrt{1-V^{2}}}{2\left(V^{2}-1\right)} \\
& =-m \pm \frac{m \sqrt{1-V^{2}}}{V^{2}-1}
\end{aligned}
$$

Since $\mathrm{Q}$ cannot be negative, we eliminate one of the roots to get

$$
\begin{aligned}
Q & =-m-\frac{m \sqrt{1-V^{2}}}{V^{2}-1} \\
& =-m+\frac{m \sqrt{1-V^{2}}}{\sqrt{1-V^{2}} \sqrt{1-V^{2}}} \\
\mathrm{Q} & =\frac{m}{\sqrt{1-v^{2}}}-m
\end{aligned}
$$

The total mass $M=m+Q ;$ hence,

$$
\mathrm{M}=\frac{m}{\sqrt{1-v^{2}}}
$$

In the theory of relativity, we compare the relationship between moving mass and rest mass during the object's motion

$$
\mathrm{M}=\frac{m}{\sqrt{1-\left(\frac{v}{c}\right)^{2}}}
$$

In the formula in this paper, $v$ is the speed of light with a maximum value of 1 . The numerical values are compared, and the relativistic mass formula $\left(\frac{v}{c}\right)$ is found to be the same. Therefore, the relativistic mass formula for $v^{2}-\left(\frac{v}{c}\right)^{2}-$ is the same. Hence, the formula in this paper is the same as the formula of relativity. Because the relativity formula has been verified for real situations, the formula presented in this paper is also validated.

This formula is convenient for use in certain calculations. For example, what is the energy of a body of mass $m=$ $1 \mathrm{~kg}$ with a velocity $V$ of $0.6 \mathrm{c}$ ? If we substitute $V=0.6 \mathrm{c}$ into the formula, we get

$$
Q=\frac{m}{\sqrt{1-V^{2}}}-m=0.25(\mathrm{~kg}) \text { and } \mathrm{M}=1.25 \mathrm{~kg} .
$$

This formula is extremely similar to the relativity-based formula and the obtained result is also the same.

\section{Discussion}

Galileo pioneered classical physics based on the concepts of time and displacement to form the basis of modern physics. The theory of relativity, based on the concepts of time and displacement, postulates the view of relative space and time and establishes the theory of relativity. For hundreds of years, physics based on this theory has made great achievements which have been validated beyond doubt. This article abandoned the concepts of displacement and time. Strictly speaking, this article is not related to existing physics, but presents a more concise and unified theory. If "all roads lead to Rome," as the saying goes, the shortest road should be of most 
interest. Of course, the investigation of the proposed perspective is as of yet limited and only beginning, requiring the dedicated work of additional researchers.

The proposed approach is able to solve relativity-related problems, and can obtain a simple, intuitive, and unified picture of physics with some further development. The basic particle, as the basic unit of mass and energy, represents the smallest possible increment of mass and energy. Mass and energy conservation thus essentially represent the conservation of these basic particles. Electrons, photons, neutrons, protons, neutrinos, and other microscopic particles are composed of these basic particles. The basic particle is also the basic foundation of force, representing the smallest increment of force in the universe. This implies force is not continuous, but consists of basic particles of force. The function of force is simply a property of basic particles. The proposed system transfers basic particles by force or radiation. A basic particle is a vector moving at the maximum velocity of the universe; however, after it transformed into a tangible substance the velocity becomes zero. The velocity of a moving object is thus a type of ratio, where the velocity direction is determined by basic particle directions. It is known that the total mass of a moving body increases with increasing velocity: this added mass is composed of basic particles provided by an external system. The concept of time is essentially academic. The nature of velocity is unrelated to time or displacement as velocity is an objective reality with a material basis. Relativity, as a mathematical model, is comprised of equations satisfying mathematical principles. Some of these, however, represent only mathematical solutions without physical meaning rather than objective physical facts.

When it is assumed that the maximum possible speed (1) is equal to the speed of light, derived results will correspond to reality. A velocity formula unrelated to displacement and time implies that the movement of an object does not depend on a reference system. For physical bodies in motion, velocity is a characteristic of the physical bodies themselves. However, these things are difficult to comprehend in the low-velocity, non-relativistic world of daily life. This situation may be likened to the old belief the sun revolved around the Earth: although this used to be indisputable, it was eventually disproved, demonstrating the true simplicity of physics and how preexisting speculations and knowledge may be false.

This raises the question: should velocity be expressed using the ratio of basic particles or displacement and time? Which has received little thought into since the pioneering works of Galileo. Today, it is perhaps time to look back and consider if the system of modern physics needs to be built on the concepts of displacement and time. Are displacement and time completely reliable concepts? If these fundamental concepts of physics have any unreliable components, then physics itself must be skewed.

Physics appears to have come to a crossroads. History has shown that humans not only can, but must reflect on the existing progress in physics, making continual corrections to the existing approach where necessary. The belief that existing theories require no further consideration is both counter-productive and unscientific. In the long course of human history, a few hundred years is a very small amount of time. Humanity is still in the infancy of scientific development. If scientists carelessly commit themselves to a single path of development in physics, attempting to describe the nature of matter in terms of space-time, this branch of science will eventually become conceptually boxed-in, limited to operating within artificial boundaries delineated by previous generations. This would be a tragedy not only for science, but for humanity. Such an approach would relegate physicists to mentally duplicating the theories of previous generations, blindly following theoretical idols without healthy skepticism or scientific spirit. There is a certain fraction of people whose commitment to their selected approach contains almost religious overtones. Scientists must be aware of the dangers of this type of thinking. Although research in physics is fraught with difficulties and challenges, the willingness to reconsider existing theories will usher in fundamental and revolutionary developments and changes.

\section{Conclusions}

This article has demonstrated the possibility of deriving an equivalent mathematical velocity expression while setting aside the concepts of displacement and time. The proposed velocity formula, better aligned with reality, allows a better understanding of the equivalence of matter and energy, which are comprised of the same type of basic particles. Why, then, is there a significant difference between matter and energy? This is because the combination of the basic particles is not the same. Basic particles are the basic unit of mass and energy, meaning mass and energy conservations are essentially the conservation of these basic particles. Electrons, photons, neutrons, protons, neutrinos, and other microscopic particles also consist of these basic particles. The basic particles are also the basic foundation of force: a basic particle force is the smallest force in the universe, implying that force is not continuous, but a basic particle force is the smallest unit of force. The total mass of a moving body increases with increasing velocities, and this added mass is composed of the basic particles provided by an external system. These basic particles are the foundation of the universe, and determine that 
physical concepts are vectors or scalars. Velocity, a vector, is the ratio between the basic particles. The concept of time is essentially academic. Although relativity equations may satisfy mathematical principles, they may represent a mathematical model with no physical meaning, not demonstrating objective physical facts. The nature of velocity has nothing to do with time or displacement, meaning velocity and space-time are unrelated. A formula was derived simply from this perspective, not relying on the complexities of relativistic space-time. Its results, however, give reason to stop and appreciate the perfection of physics. According to Occam's razor, the simplest explanation may just be the most accurate.

\section{References}

Feynman, R. P. (1982). Feynman's Lectures on Physics. Shanghai: Shanghai Science Press.

Mach, E. (1907). The science of mechanics: A critical and historical account of its development. Open court publishing Company.

\section{Copyrights}

Copyright for this article is retained by the author(s), with first publication rights granted to the journal.

This is an open-access article distributed under the terms and conditions of the Creative Commons Attribution license (http://creativecommons.org/licenses/by/4.0/). 